\title{
Phytoplankton-bacteria-protozoa interactions: a qualitative model portraying indirect effects
}

\author{
Lewi Stone \\ Yigal Allon Kinneret Limnological Laboratory, POB 345, Tiberias 14102, Israel
}

\begin{abstract}
A mathematical model is described that allows study of interactions between microbial organisms and phytoplankton when considered from a community context. In particular, the model is used to understand the paradoxical behaviour of phytoplankton which, when under nutrient stress, stimulates bacteria - the very organisms it competes with for inorganic nutrients. The model demonstrates that such behaviour, although at first sight seemingly detrimental, can be highly stable and provides phytoplankton with definite indirect advantages. Protozoa are found to be important in forming and instrumental in distributing these advantages. A general method is put forward that interprets interactions within their community context and clarifies the role of indirect effects. Such a framework makes it possible to design testable hypotheses to study further the role of microbial communities.
\end{abstract}

\section{INTRODUCTION}

In periods of nutrient limitation, phytoplankton release extracellular organic carbon (EOC), making reduced carbon available for bacterial utilization. Since both organisms compete for the same limiting inorganic nutrients, it seems paradoxical that phytoplankton should stimulate bacteria in this way (Bratbak \& Thingstad 1985). Moreover, because bacteria can be better competitors for these nutrients, the situation might well be unstable. Phytoplankton, by assisting their competitors, could suffer increasing nutrient stress, excrete more EOC, and in this vicious circle, ultimately create conditions that are detrimental to their own existence. The difficulty in understanding the paradox is part of a much broader problem: how do we determine the interaction between 2 organisms when they are embedded in a large and very complex community?

Ecologists commonly adopt a reductionist approach with which to shape their views concerning the interactions between organisms. The way in which a pair of organisms interacts is deduced from their behaviour when examined in isolation from the rest of the community. However, a full understanding of any interaction requires consideration of the organisms in their wider 'community context'. It must take into account that 2 organisms, say $A$ and $B$, can interact with one another indirectly - the interaction being mediated by other organisms in the community. Thus a change in $\mathrm{A}$ may affect another organism (say C), which in turn changes $B$.

Recent studies report that bacterivorous protozoa may influence the way in which bacteria and phytoplankton interact. These 3 organisms alone can give rise to indirect effects that need careful interpretation. For example, protozoa, being voracious bacterivores, are disadvantageous to bacteria, while bacteria compete with (and are therefore disadvantageous to) phytoplankton. Indirectly then, via the pathway of these 2 negative interactions, the protozoa must exert a positive effect on phytoplankton. The 2 organisms so form an indirect mutualism of the 'enemies' enemies' type (Boucher et al. 1982).

In the following study we present a methodology with which to analyse indirect interactions in aquatic communities and use it to re-examine the paradox presented by Bratbak \& Thingstad (1985). A mathematical model investigates the problem from a communitywide perspective and is used to find the conditions under which phytoplankton and bacteria are competitors, or instead enjoy a commensal or mutualist relationship.

Further, the model is used to determine the stability/ instability of the process whereby phytoplankton stimulates its own competitors. 


\section{DIRECT AND INDIRECT INTERACTIONS IN AQUATIC COMMUNITIES}

Fig. 1 outlines schematically the major interactions between compartments of phytoplankton $(\mathrm{P})$, bacteria (B), protozoa $(R)$, inorganic nutrients $(N)$, and zoo-

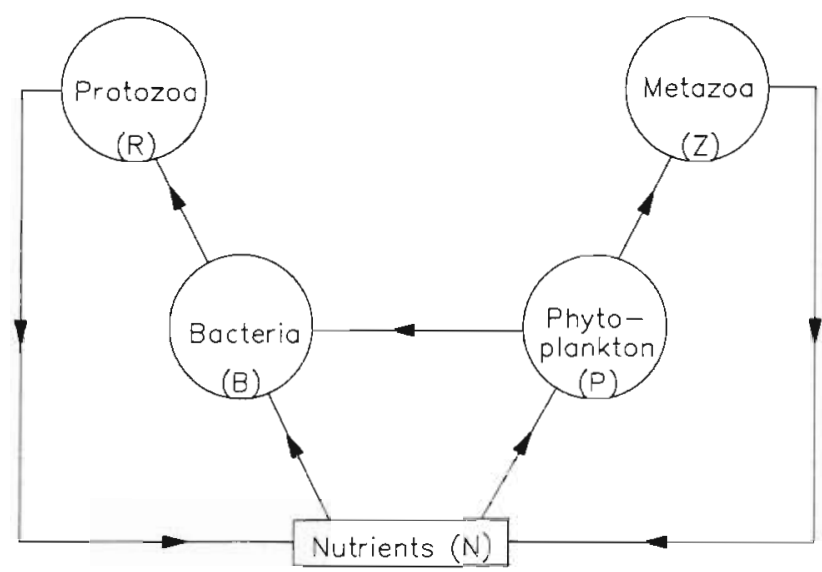

Fig. 1 Compartmental model illustrating interactions between phytoplankton $(\mathrm{P})$, bacteria $(\mathrm{B})$, protozoa $(\mathrm{R})$, inorganic nutrients (N) and zooplankton (metazoa) (Z). Arrows are used to indicate direct positive or advantageous interactions. For example, because nutrients are directly advantageous to phytoplankton, an arrow has been drawn between the 2 compartments

plankton (metazoa) (Z). The direct interaction of one compartment on another is considered to be positive or negative depending on whether it is advantageous or disadvantageous, respectively. This can be determined by considering how members of different compartments affect one another when isolated from their community setting.

A summary of all the direct interactions shown in Fig. 1 is presented in the matrix $\underline{\underline{A}}$ below, where the entry $A_{i j}$ describes the direct effect of compartment $j$ on compartment $i$. (Note that in the matrix $\underline{\underline{A}}$ all $\alpha_{1 j}>0$.) At present, we consider only the signs of the interactions $(+,-$ or 0$)$, deferring a discussion of their magnitudes to a later section. All interactions of the $(+,-)$ type (Odum 1971) (i.e. predator-prey or producer-consumer interactions) are taken to be anti-symmetric in sign. Thus if the rate at which protozoa increases from its interaction with bacteria is $A_{R B}=\alpha_{R B}>0$, then bacteria decreases at the rate $A_{A R}=-\alpha_{B R}<0$, because of protozoan grazing.

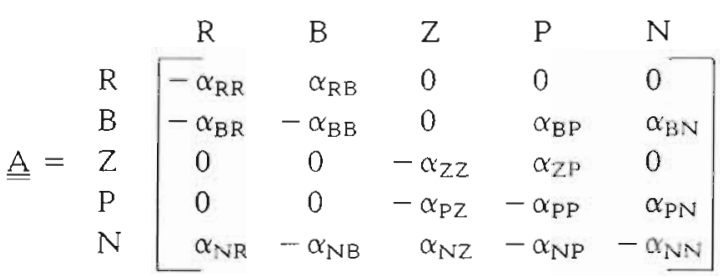

The phytoplankton-bacteria interaction is viewed as a $(+, 0)$ commensalism (Bratbak \& Thingstad 1985), since bacteria are considered here to be incapable of directly making demands on phytoplankton (e.g. by grazing) or directly affecting its growth rate. (Note carefully that the competition for nutrients between phytoplankton and bacteria cannot be considered a 'direct' interaction since it involves the third intermediary nutrient compartment.) Therefore we have taken $\mathrm{A}_{\mathrm{PB}}=0$, and $\mathrm{A}_{\mathrm{BP}}=\alpha_{\mathrm{BP}}>0$. Similarly, the relationship between protozoa and nutrients has been taken as $(+, 0)$, because protozoa contribute to the inorganic nutrient pool, but their growth-rate is not directly dependent on the availability of these nutrients. To incorporate the effect of self-interaction and intraspecific competition, the $\mathrm{A}_{\mathrm{i}}$ are taken to be negative for all compartments. (For further comments on this practice see Puccia \& Levins 1985, May 1974.) As Puccia \& Levins (1985) note, even the nutrient compartment should be modelled with a negative self-interaction term, particularly if a continuous supply of nutrient from outside the system is required.

The traditional technique of depicting a community's direct interactions only, suffers because it fails to portray the indirect effects mediated by other compartments. As an illustration, consider the way in which phytoplankton affects bacteria. Because phytoplankton release $\mathrm{EOC}$ which directly benefits bacteria, $\mathrm{A}_{\mathrm{B} \text { p }}$ must be taken as positive. However, indirectly, phytoplankton competes with bacteria for inorganic nutrients (see Fig. 1) and this is difficult to determine from the matrix A. It could be that the competition far outweighs the phytoplankton's commensalism, in which case the positive sign of $A_{B P}$ would be completely misleading as a guide to the net interaction between the 2 organisms. Moreover, both bacteria and phytoplankton are grazed upon by protozoa and zooplankton respectively, complicating the interaction further. These grazers serve to recycle nutrients taken up by bacteria and phytoplankton. Again the direct interactions yield little information as to how such processes shape the phytoplanktonbacteria interaction.

Below we present a methodology that takes into account both direct as well as indirect effects, and so allows the determination of interactions between com partments when viewed from a community context.

\section{DERIVING COMMUNITY EFFECTS BY THE INVERSE METHOD}

Consider first the very general case of a set of $M$ interacting compartments $\hat{\sigma}_{1}$ each having a biomass $N_{i}$. We suppose the equations describing their growth are in the form 


$$
\mathrm{dN}_{1} / \mathrm{dt}=\mathrm{f}_{1}\left(\mathrm{~N}_{1}, \mathrm{~N}_{2}, \ldots \mathrm{N}_{\mathrm{M}}\right), \mathrm{i}=1 \text { to } \mathrm{M}
$$

(For an outline of what this form implies, and the empirical support for it, see e.g. Yodzis 1988.)

We assume a feasible equilibrium (i.e. each compartment at equilibrium has a positive biomass $\mathrm{N}_{1}$ ) that satisfies the equations

$$
\mathrm{f}_{1}\left(\mathrm{~N}_{i}, \mathrm{~N}_{2}, \ldots \mathrm{N}_{\dot{\mathrm{M}}}^{*}\right)=0, \mathrm{i}=1 \text { to } \mathrm{M}
$$

Here, as elsewhere below, the asterisk indicates that the attached quantity is to be evaluated at equilibrium. Eq. (1) can be expanded about this equilibrium by setting

$$
N_{1}(t)=N_{i}^{*}+x_{i}(t)
$$

and we arrive at

$$
\mathrm{dx}_{\mathrm{i}} / \mathrm{dt}=\sum_{\mathrm{j}=1}^{\mathrm{M}} \mathrm{A}_{\mathrm{ij}} \mathrm{x}_{\mathrm{j}}(\mathrm{t})
$$

where

$$
A_{i j}=\left(\partial \mathrm{f}_{1} / \partial N_{j}\right)^{*}
$$

are entries of the 'community matrix' $\underline{\underline{A}}$. Any entry $A_{i j}$ describes the direct effect of the $j^{\text {th }}$ compartment $b_{j}$ (around equilibrium) on the growth rate of the $i^{\text {th }} \mathrm{com}$ partment $b_{i}$. But, as discussed above, the actual effect in the context of the whole community can be quite different. To ascertain this, we ask: how will the equilibrium $\mathrm{N}_{i}^{*}$ change if more $b_{j}$ members are introduced at a steady, continuing rate? If the $\mathrm{N}_{i}^{*}$ increases (or decreases), it seems reasonable to take this as proof that $b_{i}$ is benefitting (or suffering) from $b_{j}$.

To learn this behaviour, we carry out a 'Press' perturbation as proposed by Bender et al. (1984). We follow Yodzis (1988) in supposing that $b_{1}$ members are continually added to the community, at the rate of $\mathrm{I}_{\mathrm{j}}$ biomass units per unit area per unit time. Then Eq. (1) becomes

$$
\begin{gathered}
d N_{j} / d t=f_{j}\left(N_{1}, N_{2}, \ldots N_{M}\right)+I_{j}, \\
d N_{i} / d t=f_{1}\left(N_{1}, N_{2}, \ldots N_{M}\right), i=1 \text { to } M, i \neq j
\end{gathered}
$$

For $\mathrm{I}_{\mathrm{j}}$ small, there will be a new equilibrium at

$N_{i}\left(I_{j}\right), \ldots N_{M}\left(I_{j}\right)$, satisfying

$$
\begin{gathered}
0=\mathrm{f}_{\mathrm{j}}\left[\mathrm{Ni}_{\mathrm{i}}\left(\mathrm{I}_{\mathrm{j}}\right)_{1} \ldots \mathrm{N}_{\mathrm{M}}\left(\mathrm{I}_{\mathrm{j}}\right)\right]+\mathrm{I}_{\mathrm{j}} \\
0=\mathrm{f}_{\mathrm{i}}\left[\mathrm{Ni}_{\mathrm{i}}\left(\mathrm{I}_{\mathrm{j}}\right)_{1} \ldots \mathrm{N}_{\mathrm{M}}\left(\mathrm{I}_{\mathrm{j}}\right)\right]_{,} \mathrm{i}=1 \text { to } \mathrm{M}, \mathrm{i} \neq \mathrm{j}
\end{gathered}
$$

Differentiating Eq. (5) with respect to $I_{j}$, we find, using Eq. (3),

$$
\begin{gathered}
-1=\sum_{k} A_{j k} \partial N_{k}\left(I_{j}\right) / \partial I_{j,} \\
0=\sum_{k} A_{i k} \partial N_{k}\left(I_{j}\right) / \partial I_{j}, i=1 \text { to } M, i \neq j
\end{gathered}
$$

Solving Eq. (6) for the unknown derivatives, we obtain

$$
\partial \mathrm{N} ;\left(\mathrm{I}_{\mathrm{J}}\right) / \partial \mathrm{I}_{\mathrm{j}}=-\left(\underline{\underline{A}}^{-1}\right)_{\mathrm{i}_{1}}
$$

The left side here gives the direction in which the $b_{i}$ biomass, after settling down, responds to a steady influx into $b_{1}$. This is the interaction in a community context that we seek, and Eq. (7) shows that it is described by the $(i, j)^{\text {th }}$ entry of the inverse of the matrix $(-\underline{\underline{A}})$. We therefore define (following Stone 1988) the matrix of community effects to be

$$
\underline{\underline{E}}=-\underline{\underline{A}}^{-1}=\left(e_{1 \jmath}\right)
$$

To determine the qualitative effect of compartment $b_{j}$ on $b_{i}$ in a community context, we need only look at the sign of $\mathrm{e}_{i j}$.

(1) if $e_{i j}>0$, then compartment $b_{j}$ is advantageous to $b_{1}$. The interaction is therefore considered as advantageous in a community context.

(2) If $e_{i j}<0$, then compartment $b_{j}$ is disadvantageous to $b_{1}$ when viewed from a community context.

\section{COMPARISON OF THE INVERSE METHOD WITH LOOP ANALYSIS}

Loop analysis has been a tool employed in a number of studies in order to interpret indirect interactions (Levins 1973, Lane \& Levins 1977. Puccia \& Levins 1985). Levins (1975) designed a comprehensive framework for such analyses in which all the feedback loops (or indirect pathways) of various 'lengths' and 'levels', found within the interaction matrix, were enumerated. However, loop analysis assigns only the values $+1,-1$ or 0 to an interaction coefficient. This restriction on the coefficients becomes a severe limitation. Levins (1973) warns that it is quite possible to obtain ambiguous or even wrong results when the magnitudes of interactions are ignored in this way. Despite this limitation, the method has proved to be a useful explorative tool, but unfortunately, as Platt (1981) makes evident, has hardly been utilized for studies of marine environments.

The inverse method is in fact an extension of loop analysis (see Stone \& Roberts unpubl. for an examination and comparison of the 2 approaches). As well as being simpler to implement, the inverse method allows for the magnitudes of interactions. Even though interaction coefficients are difficult and sometimes impossible to calculate accurately, the inverse method has the advantage of portraying effects introduced by changes in interaction strengths. It is necessary to point out that results considered ambiguous by loop analysis can be understood, and are expected to arise, when the magnitudes and changes of interactions are taken into account. 


\section{PHYTOPLANKTON-BACTERIA-PROTOZOA INTERACTIONS}

With the above framework, it is now possible to elucidate the community effects for the compartmental structure portrayed in Fig. 1. We begin by examining the hypothetical community matrix $\underline{\underline{A}}$ given below. In this example, the coefficients $A_{\mathrm{l} j}$ were chosen for demonstration purposes only and (as discussed in more detail below) fell within a range that ensured the system to be stable.

It was assumed that the microbial loop recycled nutrients at a relatively rapid rate (Goldman 1984) and therefore the coefficients associated with this recycling were set with relatively large magnitudes (e.g. $\mathrm{A}_{\mathrm{PB}}=$ $\left.A_{N P}=0.6\right)$. Further it was assumed bacterial uptake of nutrients was greater than that of phytoplankton $\left(A_{B N}\right.$ $\left.=0.6, A_{P N}=0.5\right)$. All self-interaction terms $A_{u}$ were scaled to unity (following May 1974). The community effects matrix $\underline{\underline{E}}=-\underline{\underline{A}}^{-1}$ was then determined by computer using a standard matrix inversion routine.

\begin{tabular}{l|rrrrr}
\multicolumn{1}{c}{$\mathrm{R}$} & $\mathrm{B}$ & \multicolumn{1}{c}{$\mathrm{Z}$} & $\mathrm{P}$ & \multicolumn{1}{c}{$\mathrm{N}$} \\
$\mathrm{R}$ & -1.0 & 0.6 & 0.0 & 0.0 & 0.0 \\
$\mathrm{~B}$ & -0.6 & -1.0 & 0.0 & 0.1 & 0.6 \\
$\mathrm{Z}$ & 0.0 & 0.0 & -1.0 & 0.2 & 0.0 \\
$\mathrm{P}$ & 0.0 & 0.0 & -0.2 & -1.0 & 0.5 \\
$\mathrm{~N}$ & \multicolumn{1}{c}{0.6} & -0.6 & 0.2 & -0.5 & -1.0 \\
\hline
\end{tabular}

\begin{tabular}{|c|c|c|c|c|c|}
\hline & $\mathrm{R}$ & B & Z & $\mathrm{P}$ & $N$ \\
\hline $\mathrm{R}$ & .89 & .40 & .05 & .06 & .21 \\
\hline B & -.19 & .67 & .09 & -.09 & .36 \\
\hline Z & .05 & -.01 & .98 & .16 & .07 \\
\hline $\mathrm{P}$ & .25 & -.06 & -.09 & .80 & .36 \\
\hline $\mathrm{N}$ & .53 & -.13 & .22 & -.34 & .75 \\
\hline
\end{tabular}

The community effects matrix immediately makes clear the following points:

(1) One sees that phytoplankton and bacteria suffer from the presence of each another since both $e_{\mathrm{BP}}=$ -0.09 and $e_{\mathrm{pB}}=-0.06$ are negative quantities. This is to be expected because the 2 organisms compete for common nutrients. However, the matrix $\underline{\underline{A}}$ of direct interactions fails to reveal this competition

(2) In fact $e_{\mathrm{BP}}<e_{\mathrm{PB}}$, indicating that bacteria suffer from the presence of phytoplankton more than phytoplankton suffers from the presence of bacteria. It thus appears that phytoplankton is a superior competitor for nutrients. This result is rather surprising, since the coefficients of matrix $\underline{\underline{A}}$ indicate that the rate of bacterial uptake of nutrients is greater than that for phytoplankton - (falsely) suggesting that for this community, bacteria have the superior competitive ability. Further, that bacteria should suffer greater losses than phytoplankton is even more surprising when one considers that the latter organism is actually stimulating the former $\left(A_{B P}=0.1\right)$.

(3) The matrix E $\underline{\underline{E}}$ also makes clear that the presence of protozoa benefits phytoplankton (as discussed in the 'Introduction') since $\mathrm{e}_{\mathrm{PR}}=0.25$; a fact not obvious from $\underline{\underline{A}}$ in which $\mathrm{A}_{\mathrm{PR}}=0$.

\section{GENERAL ANALYSIS}

Because the interaction coefficients in the matrix $\underline{\underline{A}}$ are difficult to obtain and are highly specific to the particular system being modelled, we proceed by calculating E algebraically (see Appendix 1) for the completely general case. By proceeding this way it becomes possible to interpret the non-intuitive results discussed above. In order to simplify the problem, but without losing generality, the self-interaction terms are again scaled to have unit magnitude so that $A_{i i}=-1$ (following May 1974). While this assumption is not necessary for our study, it makes the task of focusing explicitly on intercompartmental interactions more amenable.

\section{Protozoan interaction with phytoplankton $\mathrm{e}_{\mathrm{PR}}$}

Appendix 1 demonstrates that the effect of the protozoa on the phytoplankton, when viewed from a community-wide context, is given by

$$
\begin{aligned}
e_{P R}= & \alpha_{P N} \alpha_{N R}+\alpha_{P N}\left(-\alpha_{N B}\right)\left(-\alpha_{B R}\right) \\
& =\alpha_{P N} \alpha_{N R}+\alpha_{P N} \alpha_{N B} \alpha_{B R}
\end{aligned}
$$

But, since all $\alpha_{i j}$ are positive, $e_{\mathrm{PR}}>0$ under all conditions. Therefore protozoa is always advantageous to phytoplankton when viewed from a community context. The positive effect arises from 2 pathways of indirect effects, as the right hand side of Eq. (9) shows, and these are discussed separately below.

(1) The term $\alpha_{\mathrm{PN}} \alpha_{\mathrm{NR}}$ indicates the pathway by which protozoa recycle organic material to the inorganic nutrient pool where it is then once more taken up by phytoplankton. Visually we could portray the flows as follows

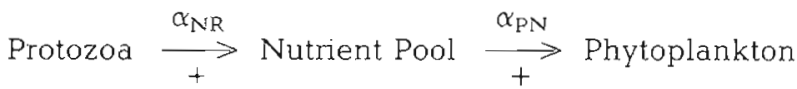

(2) The contribution from the term $\alpha_{P N}\left(-\alpha_{N B}\right)\left(-\alpha_{B R}\right)$ depicts the pathway from the protozoa compartment through to the phytoplankton compartment, thus 


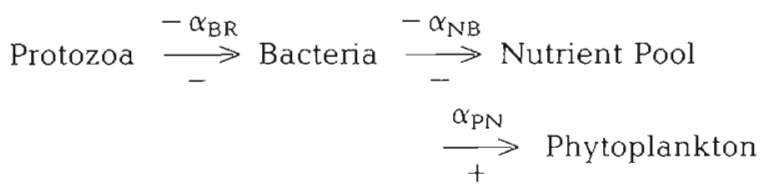

There are 2 negative interactions (the effect of the protozoa on the bacteria, and the effect of the bacteria on the nutrient pool) which, when taken together, form a net positive interaction. An increase in protozoa will decrease bacteria (reducing bacterial nutrient demands), and therefore make more nutrients available for phytoplankton uptake. For this reason, as Berman et al. (1987), Caron et al. (1988) and Sherr et al. (1988) have argued, the grazing activity of protozoa may be an important source of nutrients for primary producers, particularly under limiting conditions. As mentioned in the introduction, protozoa and phytoplankton form an indirect mutualism of the 'enemies' enemies' type.

The above analysis demonstrates that even though phytoplankton and protozoa do not interact directly, they exert on one another a positive effect that comes about purely as a result of indirect effects. These indirect effects set the stage for coevolutionary adaptions. The cost of stimulating bacterial competitors might well be worth the indirect advantages offered by the protozoa. This is but another example in ecology of turning 'competitors into accomplices' (Hay 1986) in order to reap the benefits of another party, in this case the protozoa.

\section{Bacteria interaction with phytoplankton $e_{\mathrm{PB}}$}

To determine the effect bacteria has upon phytoplankton, when viewed from a community context, we examine $e_{\mathrm{PB}}$. Using the method given in Appendix 1, it can be shown that

$e_{\mathrm{PB}}=\alpha_{\mathrm{PN}} \alpha_{\mathrm{NR}} \alpha_{\mathrm{RB}}+\alpha_{\mathrm{PN}}\left(-\alpha_{\mathrm{NB}}\right)=\alpha_{\mathrm{PN}}\left(\alpha_{\mathrm{NR}} \alpha_{\mathrm{RB}}-\alpha_{\mathrm{NB}}\right)$

Again consider the 2 contributions separately.

(1) The term $\alpha_{\mathrm{PN}} \alpha_{\mathrm{NR}} \alpha_{\mathrm{RB}}$ is a positive feedthrough pathway (being the product of 3 positive interactions) flowing from

$$
\begin{aligned}
\text { Bacteria } \stackrel{\alpha_{\mathrm{RB}}}{\longrightarrow} \text { Protozoa } \stackrel{\alpha_{\mathrm{NR}}}{\rightarrow} \text { Nutrients } \\
\stackrel{\alpha_{\mathrm{PN}}}{\longrightarrow} \text { Phytoplankton }
\end{aligned}
$$

An increase (or decrease) in bacteria passes through the loop and so increases (or decreases) phytoplankton. (2) In contrast, the contribution due to $\alpha_{P N}\left(-\alpha_{\mathrm{NB}}\right)$ is negative (being the product of a positive and a negative interaction) and flows from

$$
\text { Bacteria } \stackrel{-\alpha_{N B}}{\longrightarrow} \text { Nutrients } \underset{+}{\stackrel{\alpha_{N P}}{\longrightarrow}} \text { Phytoplankton }
$$

Eq. (10) shows clearly how the presence of the protozoa alleviates the competitive pressure of bacteria on phytoplankton. The more the bacteria that are grazed by protozoa (i.e. the greater $\alpha_{R B}$ ), and the more the protozoa remineralize nutrients (i.e. the greater $\alpha_{N R}$ ), then the less negative is the effect of bacteria on phytoplankton $\left(e_{P B}\right)$. Hence rapid bacterivory would result in shifting the competition for inorganic nutrients in favor of phytoplankton, concurring with prediction of Sherr et al. (1988)

We see from Eq. (10) that the possibility exists for bacteria to have a net positive effect on phytoplankton when the positive feedthrough term is greater than the negative effects. Thus

$$
\mathrm{e}_{\mathrm{PB}}>0 \text { when } \quad \alpha_{\mathrm{NR}} \alpha_{\mathrm{RB}}>\alpha_{\mathrm{NB}}
$$

Effectively this means that if the recycling rates (from bacteria to protozoa, and protozoa to nutrients) are fast enough, with respect to the rate at which bacteria take up inorganic nutrients, then bacteria can have a net positive effect on their own direct competitor - phytoplankton. Indeed this event has been noted in several studies (Swift 1980, Brand 1986) but has, however, been difficult to explain.

\section{Phytoplankton interaction with bacteria $e_{B P}$}

It is also possible to define the conditions under which phytoplankton affects bacteria advantageously. It can be found that

$$
\begin{aligned}
e_{B P} & =\alpha_{B N} \alpha_{N Z} \alpha_{Z P}+\alpha_{B N}\left(-\alpha_{N P}\right)+\alpha_{B P} \\
& =\alpha_{B N}\left(\alpha_{Z P} \alpha_{N Z}-\alpha_{N P}\right)+\alpha_{B P}
\end{aligned}
$$

Thus phytoplankton is advantageous to bacteria if

$e_{\mathrm{BP}}>0$, when $\quad \alpha_{\mathrm{BN}} \alpha_{\mathrm{NZ}} \alpha_{\mathrm{ZP}}+\alpha_{\mathrm{BP}}>\alpha_{\mathrm{BN}} \alpha_{\mathrm{NP}}$

Suppose that the phytoplankton stimulation was negligible so that $\alpha_{\mathrm{BP}}=0$. We would then obtain

$$
\mathrm{e}_{\mathrm{BP}}>0, \quad \text { when } \quad \alpha_{Z \mathrm{P}} \alpha_{\mathrm{NZ}}>\alpha_{\mathrm{NP}}
$$

The above condition makes clear that unless the phytoplankton-zooplankton-nutrient recycling pathway is very active, $e_{\mathrm{BP}}<0$ and bacteria will generally suffer from the presence of phytoplankton. However, the situation could well reverse for the case when $\alpha_{B P}>$ 0 . If the stimulation were large enough then there may be commensalism. The positive effects of bacterial stimulation and zooplankton recycling could outweigh competition for nutrients, and bacteria would then be advantaged from the presence of phytoplankton. 
Seen from a different perspective, suppose the recycling as carried out by the microbial pathways is faster than zooplankton recycling (a realistic assumption; Goldman 1984), so that the following inequality holds

$$
\begin{aligned}
\alpha_{P N} \alpha_{N R} \alpha_{R B}>\alpha_{B N} \alpha_{N Z} \alpha_{Z P,} & \text { and therefore } \\
\alpha_{P N} \alpha_{N R} \alpha_{R B}=\alpha_{B N} \alpha_{N Z} \alpha_{Z P}+k & \text { where } k>0
\end{aligned}
$$

Note that the above may be true even though $\alpha_{\mathrm{BN}}>\alpha_{\mathrm{PN}}$ (i.e. bacteria uptake of nutrients is greater than that of phytoplankton). The direct producer-consumer interactions may be taken to have an antisymmetric structure, and it is plausible to set $\alpha_{\mathrm{PN}} \alpha_{\mathrm{NB}}=\alpha_{\mathrm{BN}} \alpha_{\mathrm{NP}}$ (refer to Appendix 2). Eqs. (10), (12) and (14) then give

$$
\mathrm{e}_{\mathrm{BP}}=\mathrm{e}_{\mathrm{PB}}+\alpha_{\mathrm{BP}}-\mathrm{k}_{,} \quad \text { where } \mathrm{k}>0
$$

Again consider the case when there is no phytoplankton stimulation of the bacteria so that $\alpha_{\mathfrak{B D}}=0$. Then from Eq. (15) we see,

$$
e_{\mathrm{BP}}<\mathrm{e}_{\mathrm{PB}}
$$

The above relation implies bacteria suffer more from phytoplankton than phytoplankton suffer from bacteria. Thus, in the community-wide context, phytoplankton can be stronger competitors than bacteria. Under these conditions, phytoplankton can afford to stimulate their bacteria competitors. Paradoxically, this can be true even if bacteria are better direct competitors for nutrients than phytoplankton $\left(\alpha_{N B}<\alpha_{N P}\right)$.

\section{STABILITY OF THE MODEL}

The model presented here can be used to explore the stability of the commensal/competitive relationship between phytoplankton and bacteria. It appears that phytoplankton, when stressed by lack of nutrients, stimulates its (bacterial) competitors by releasing EOC, and thereby reduces its own nutrient supply. Such a process seems inherently unstable because any induced stress encourages more EOC release, which stimulates bacteria further, reduces more of the available nutrients and thus creates even greater stress (Bratbak \& Thingstad 1985, Sherr et al. 1988). Described in this way, the phytoplankton stimulation could be seen as a means of supplying positive feedback to bacteria, resulting in spiralling bacterial growth but rapidly diminishing phytoplankton nutrient supply. If this interpretation were true, one could view the positive feedback as unstable and highly detrimental.

To understand the effects of the positive feedback in more detail, the model's global stability has been examined in Appendix 3. A model that possesses global stability has the ability to recover to its equi- librium state no matter how damaging the perturbations it experiences (Chesson \& Case 1986). This is far stronger than (the commonly studied) local stability which predicts recovery only for 'small' perturbations about equilibrium. It was found that the model's parameters $\alpha_{B P}$ (which controls the phytoplankton stimulation of bacteria), $\alpha_{\mathrm{NZ}}$ and $\alpha_{\mathrm{NR}}$ (which control nutrient recycling) determine the model's global stability. Our analysis showed this stability to result as long as the following 2 constraints are satisfied

$$
\alpha_{\mathrm{BP}}<2 \text { and }\left(\alpha_{\mathrm{NZ}}^{2}+\alpha_{\mathrm{NR}}^{2}\right)<4
$$

In fact this parameter range guaranteeing global stability is quite broad. Consider for example the bacteria compartment, which has the ratio $\mathrm{R}_{\mathrm{B}}$ for the sum of its intercompartmental interactions to its intracompartmental (or self) interaction. If all bacteria's intercompartmental interactions were of magnitude $2, R_{B}=6$. But then because $\alpha_{B P}=2$, the model would be at the edge of global stability (though it may well be locally stable for $\alpha_{\mathrm{BP}}>2$ ). Intercompartmental interactions would then be some 6 times higher than intracompartmental effects indicating a regime where the community has quite a large (and perhaps unrealistic) influence on the bacteria compartment. Because the model is globally stable over this wide parameter range, it seems likely that only in rare circumstances would the phytoplankton-bacteria interaction cause instability.

These results have important and general ecological consequences. In particular, evidence of any positive feedback is not sufficient reason to argue that it causes instability or that its occurrence is paradoxical. Positive feedback can be an integral process in stable systems.

\section{DISCUSSION}

Bratbak \& Thingstad (1985) found difficulty in resolving the paradox of phytoplankton stimulating its own competitor-bacteria. They suggested further insight might be obtained by the inclusion of higher trophic levels into their model. However, they argued that it was not immediately clear how this could be of advantage to the phytoplankton since the bacteria and the predator chain would necessarily tie up mineral nutrients in their biomass'.

In the model discussed here, it was indeed found that by including protozoa, and allowing for their ability to graze bacteria and recycle nutrients, many aspects of the paradox could be resolved. The presence of the protozoa gives rise to a number of indirect interactions which are advantageous to phytoplankton. Perhaps the advantage most immediately clear is the indirect mutualism the protozoa forms with phytoplankton of the 'enemies' enemies' type, that is mediated by bacteria. 
It was also shown that, because the protozoa are relatively rapid grazers and recyclers, then indirectly, they can alleviate the competitive pressure of bacteria on phytoplankton by providing a supply of nutrients to the phytoplankton that would otherwise be absent. For this reason alone, there could be definite payoffs for phytoplankton should it stimulate bacteria. Even more unusual is the possibility that the presence of the protozoa can create conditions in which the bacteria are advantageous to their phytoplankton competitors - so that an increase in bacteria will cause an increase in the phytoplankton equilibrium.

While many studies are reporting bacteria to be better competitors than phytoplankton for inorganic nutrients, it should be kept in mind that this conclusion is usually derived from a study of relative nutrient uptakes of the 2 organisms when considered in isolation, or when abstracted from their natural setting Also, investigations are sometimes carried out over inappropriately chosen time scales so that the community may be given little time to respond, and its effects are therefore not observed. However, in order to determine the real interaction between 2 organisms, it is crucial that correct consideration be given to interactions with their surrounding community. The model made clear that even if bacteria have greater facility for nutrient uptake, phytoplankton can have the superior competitive ability when the indirect effects introduced by protozoan recycling are strong. These circumstances would place phytoplankton in a position where it could afford to stimulate its (weaker) competitors. As the model bears out, this so-called 'paradoxical' behaviour can very often be a stable process.

The community we have modelled has not incorporated pathways that may be quite important in some ecosystems. For example, in some aquatic environments, protozoa are known to graze phytoplankton directly. However, the framework presented here could be used to explore the effects of any such extra pathways. In this initial study it was demonstrated that, even in a simple community of 2 compartments, indirect effects have complex and important ramifications that cannot be ignored.

It has been suggested that positive indirect interactions, similar to the ones reported here, may have evolutionary implications whereby organisms adapt and also modify their community in ways that might loop back advantageously upon themselves (Wilson 1987). As yet there is insufficient evidence, but recent investigations suggest that the indirect effects of microbial components appear to lead to coevolutionary behaviour (Wood \& Van Valen 1989). The methodology presented in this study should prove a useful tool that allows the determination of the indirect pathways and the evolutionary pressures they might exert. In doing so, it can provide an important guide for the formulation of testable hypotheses with which to analyse complex communities.

Acknowledgements. I thank Michelle Wood, Rick Weisburd Werner and Sarah Eckert for encouragement and valuable discussions. I am most grateful to Tom Berman and Bonnie Azoulay for assistance in planning, as well as reviewing the manuscript.

\section{APPENDIX 1}

The following method can be used to determine the inverse of the matrix $(-\underline{\underline{A}})$. Define $C_{i j}$ to be the cofactor of the element $(\underline{\underline{A}})_{i j}$. Thus

$$
\mathrm{C}_{\mathrm{ij}}=(-1)^{i+\mathrm{i}}\left|\underline{\underline{\mathrm{A}_{(i)}}}\right|
$$

where $\underline{\underline{A}}_{(i)}$ is the $(M-1) \times(M-1)$ submatrix which results from

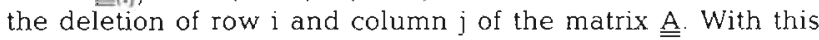
notation,

$$
e_{i j}=\left(-\underline{\underline{A}}^{-1}\right)_{1 j}=\delta C_{j i} \text { where } \delta=\frac{1}{|-\underline{\underline{A}}|}(\text { Bowman 1964) }
$$

In Appendix 3 we derive conditions under which the model is globally stable. In these circumstances, $|-\underline{A}|>0$ (May 1974, p. 195) and thus $\delta$ is positive. Since all components $\left(-\underline{\underline{A}}^{-1}\right)_{\text {ij }}$ have a common positive factor $\delta$, then for the purposes of this study it is possible, without any loss of generality, to take the scaling

$$
e_{i j}=C_{j 1}
$$

As an example, we calculate $e_{p R}$.

$$
\begin{aligned}
e_{P R}=C_{R P} & =(-1)^{1+4}\left|\begin{array}{cccc}
-\alpha_{B R} & -1 & 0 & \alpha_{B N} \\
0 & 0 & -1 & 0 \\
0 & 0 & -\alpha_{P Z} & \alpha_{P N} \\
\alpha_{N R} & -\alpha_{N B} & \alpha_{N Z} & -1
\end{array}\right| \\
& =\alpha_{P N N} \alpha_{N R}+\alpha_{P N}\left(-\alpha_{N B}\right)\left(-\alpha_{B R}\right)
\end{aligned}
$$

\section{APPENDIX 2}

It is necessary to comment on the comparison of the magnitudes of the community effect $e_{B P}$ with $e_{P B}$ as discussed in the text. While the mathematical formulation was developed chiefly to determine the sign of the community effects $e_{i j}$, it still provides a means by which to examine and compare the magnitudes of the $e_{i j}$. In order to carry out such an analysis, we note that it is only meaningful to make comparisons of the net per capita community effects

$$
\varepsilon_{i j}=e_{1 j} / N_{i}
$$

Here $\varepsilon_{\mathrm{ij}}$ is the net per capita response of compartment $b$ caused by a steady influx into $b_{j}$.

Any precise comparison also requires that the model (Eq. 1) be specified more fully. Thus, we fix our ideas to the simplest non-linear model of a community, the Generalized LotkaVolterra' model

$$
\mathrm{dN}_{\mathrm{l}} / \mathrm{dt}=\mathrm{N}_{1}\left(\mathrm{r}_{1}+\sum \beta_{3 j} \mathrm{~N}_{\mathrm{j}}\right) \quad \mathrm{i}=1 \text { to } \mathrm{M}
$$

which is itself a first-order approximation to a very general set of equations (May 1974). Here $r_{1}$ are the intrinsic increase rates, and $\beta_{13}$ are the interaction coefficients which are entries of the matrix $\underline{\underline{B}}$. Note that $\beta_{1 j}$ have an antisymmetric structure if they are of the $(+,-)$ type, so that the predator's (consumer's) 
gains are proportional to the prey's (producer's) losses. As May (1974, p. 51) discusses, this requires that all $(+,-)$ interactions satisfy

$$
\beta_{13}=-\beta_{1 \mathrm{i}}
$$

The equilibrium populations of $(\mathrm{A} 2.1)$ are given by

$$
N_{i}^{*}=-\sum\left(\underline{B}^{-1}\right)_{j j} r_{j} \quad i=1 \text { to } M
$$

and the 'community matrix' $\underline{\underline{\mathrm{A}}}$ (Eq. 3) is given by (May 1974. p. 22)

$$
A_{i j}=\left(\partial f_{1} / \partial N_{j}\right)^{*}=N_{i} \beta_{i j}
$$

The community effects matrix $\underline{\underline{E}}=-\underline{\underline{A}}^{-1}=-\underline{\underline{B}}^{-1} \underline{\underline{D}}^{-1}$ where the matrix $\underline{\underline{D}}=\operatorname{diag}\left(\mathrm{N}_{i}, \mathrm{~N}_{2}, \ldots \mathrm{N}_{M}^{*}\right)$. Thus

$$
e_{1,}=-\left(\underline{\underline{B}}^{-1}\right)_{\mathrm{ij}} / \mathrm{N}_{\mathrm{j}}^{\mathrm{j}} \quad \text { so that } \quad \varepsilon_{\mathrm{ij}}=-\left(\underline{\underline{B}}^{-1}\right)_{1]} /\left(\mathrm{N}_{\mathrm{i}}^{\mathrm{N}} \mathrm{N}_{\mathrm{i}}\right)
$$

This last equation shows that $\varepsilon_{i j}$ are symmetric in $\mathrm{N}_{j}$ and $\mathrm{N}_{j}$. Thus to compare $\varepsilon_{\mathrm{BP}}$ with $\varepsilon_{\mathrm{PB}}$ requires only a comparison of $-\left(\underline{\underline{B}}^{-1}\right)_{B P}$ with $-\left(\underline{\underline{B}}^{-1}\right)_{P B}$ where the $(+,-)$ interactions contained in $\underline{B}$ are anti-symmetric. Equivalently, as we have done in the text, one could simply compare $e_{B P}$ with $e_{P B}$ and assume that the $\alpha_{i 1}$ interactions of the $(+,-)$ type are antisymmetric.

\section{APPENDIX 3}

The conditions are found for the global stability of Eq. (1). A system that possesses global stability will return to equilibrium no matter what size of perturbation disturbs it and is thus far better able to cope with disturbances than if it were only locally stable. It is assumed that all $(+,-)$ interactions have an antisymmetric structure so that the predator's (consumer's) gains are proportional to the prey's (producer's) losses (May 1974). Here we suppose the community matrix has entries $A_{i j}=-A_{j i}$ for all of the $(+,-)$ interactions. While it is known that this assumption does not always hold directly, the conclusions arrived at can be equally appropriate for investigations of stability (as May [1974] p. 50-51 demonstrates mathematically) ${ }^{\text {a }}$.

Let $\underline{\underline{A}}^{\top}$ be the transpose of the matrix $\underline{\underline{A}}$ and define the symmetric matrix $\underline{\underline{G}}=0.5\left(\underline{\underline{A}}+\underline{\underline{A}}^{\mathrm{T}}\right)$. Then Eqs. (1) or (A2.1) are globally stable if $\underline{\underline{G}}$ is negative definite (Goh 1977, Berman \& Plemmons 1979, i.e. if all eigenvalues $\lambda_{i}$ of $\underline{\underline{G}}$ (which alre real) are negative

The eigenvalues can be found by evaluating the determinant

$$
|\underline{\underline{G}}-\lambda \underline{\underline{I}}|=0 \text {, where } \underline{\underline{I}} \text { is the identity matrix. }
$$

Some algebra shows the eigenvalues to be

$$
\gamma=-1,-1 \pm \frac{1}{2} \alpha_{\mathrm{BPt}}-1 \pm \frac{1}{2} \sqrt{\left(\alpha_{\mathrm{NZ}}^{2}+\alpha_{\mathrm{NR}}^{2}\right)}
$$

Thus the model is globally stable when.

$$
\alpha_{\mathrm{BP}}<2, \text { and }\left(\alpha_{N Z}^{2}+\alpha_{N R}^{2}\right)<4
$$

\footnotetext{
"For the important Lotka-Volterra model (A2.1), which is itself a first-order approximation to a very wide class of models, interactions of the $(+,-)$ type are glven by $(\mathrm{A} 2.2)$ (as discussed in Appendix 2). Now make the substitution $A_{1 j}=\beta_{i j}$. According to the analysis in this Appendix, global stability would require the matrix $\underline{G}_{2}=\underline{D} \mathrm{G}$ (where $\left.\underline{\underline{D}}=\operatorname{diag}\left(N_{i}, N_{2}, \ldots N_{M}\right)\right]$ to be negative definite. However, for a feasıble equilibrium (where all $\mathrm{N}_{i}>0$ ), the necessary and sufficient condition for global stablity is still that the matrix $G$ be negative definite (Goh 1977. Berman \& Plemmons 1979). Thus the stability criteria reduces to that given above (A3.1)
}

\section{LITERATURE CITED}

Bender, E. A., Case, T J., Gilpin. M. E. (1984). Perturbation experiments in community ecology: theory and practice. Ecology 65: 1-13

Berman, T., Nawrocki, M., Taylor, G. T., Karl, D. (1987). Nutrient flux between bacteria, bacterivorous nanoplanktonic protists and algae. Mar. microb. Food Webs 2 (2): $69: 82$

Berman, A., Plemmons, R. J. (1983). Nonnegative matrices in the mathematical sciences. Academic Press, New York

Boucher, D. H., James, S., Keeler, K. H. (1982). The ecology of mutualism. Ann. Rev. Ecol. Syst. 13: 315-347

Bowman, F. (1964). An introduction to determinants and matrices. English University Press, London

Bratbak, G., Thingstad, T F. (1985). Phytoplankton-bacteria interactions: an apparent paradox? Analysis of a model system with both competition and commensalism. Mar Ecol. Prog. Ser. 25: 23-30

Brand, L. E. (1986). Nutrition and culture of autotrophic ultraplankton and picoplankton. In: Platt, T., Li, W. K. W. (eds.) Photosynthetic picoplankton. Can. Bull. Fish. Aquat. Sci. 214: 205-234

Caron, D. A., Goldman, J. G., Dennett, M. R. (1988). Experimental demonstration of the roles of bacterivorous protozoa in plankton nutrient cycles. Hydrobiologia 159 $27-40$

Chesson, P. L., Case, T J. (1986). Overview: nonequilibrium community theories: chance, variability, history, and coexistence. In: Diamond, J., Case, T. J. (eds.) Community ecology, Harper and Row, New York, p. 229-239

Goh, B. S. (1977). Global stability in many species systems Am. Nat. 111. 135-143

Goldman. J. C. (1984). Oceanic nutrient cycles. In: Fasham, M. J. R. (ed.) Flows of energy and materials in marine ecosystems, Plenum Press, New York, p. 137-170

Hay, M. E. (1986). Associational plant defences and the maintenance of species diversity: turning competitors into accomplices. Am. Nat. 128: 617-641

Lane, P. A., Levins, R. (1977). The dynamics of aquatic systems. 2. The effects of nutrient enrichment on model plankton communities. Limnol. Oceanogr. 22: 454-471

Levins, R. L. (1973). Discussion paper: the qualitative analysis of partially specified systems. Ann. N. Y. Acad. Sci. 231: $123-138$

Levins, R. (1975). Evolution in communities near equilibrium. In: Cody, M. L., Diamond, J. M. (eds.) Ecology and evolution of communities. Belknap Press, Harvard University, p. $16-50$

May, R. M. (1974). Stability and complexity in model ecosystems. University Press, Princeton

Odum, E. P. (1971). Fundamentals of ecology, 3rd edn. W B. Saunders Co., Philadelphia

Platt, T. (1981). Loop analysis. In: Platt, T., Mann, K. H., Ulanowicz, R. E. (eds.) Mathematical models in biological oceanography. UNESCO Press, Paris, p. 64-65

Puccia, C. J., Levins, R (1985). Qualitative modeling of complex systems - an introduction to loop analysis and time averaging. Harvard University Press, Cambridge

Sherr, B. F., Sherr, E. B., Hopkinson, C. S. (1988). Trophic interactions within pelagic microbial communities: Indications of feedback regulation of carbon flow. Hydrobiologia 159: $19-26$

Stone, L. (1988). Some problems of community ecology: Processes, patterns and species persistence in ecosystems. Ph. D. thesis, Monash University, Australla

Swift, D. G. (1980). Vitamins and phytoplankton growth. In: 
Morris, I. (ed.) The physiological ecology of phytoplankton. Univ. of California Press, Berkeley, p. 329-368

Wilson, D. S. (1986). Adaptive indirect effects. In: Dlamond, J., Case, T. J. (eds.) Community ecology, Harper and Row, New York, p. $437-444$

This article was presented by Professor T. Berman, Tiberias, Israel
Wood, A. M., Van Valen, L. M. (1990). Paradox lost? On the release of energy-rich compounds by phytoplankton. Mar microb, Food. Webs (in press)

Yodzis, P. (1988). The indeterminacy of ecological interactions as perceived through perturbation experiments. Ecology 69 (2): $508-512$

Manuscript first received: October 12, 1989

Revised version accepted: Masch 20, 1990 